\title{
Grupos de Soporte Nutricional en Colombia: resultados del nutritionDay 2011-2016
}

\author{
Nutrition support teams in Colombia results from the \\ nutritionDay 2011-2016
}

\author{
Diana Cárdenas ${ }^{\text {** }}$, Josef Kling², Gustavo Díaz', Charles Bermúdez ${ }^{3}$.
}

Recibido: 20 de mayo de 2018. Aceptado para publicación: 27 de Junio de 2018 https://doi.org/10.35454/rncm.v1n2.044

\begin{abstract}
Resumen
El cuidado nutricional a través de grupos interdisciplinarios de soporte nutricional y bajo estándares y guías de manejo ha demostrado mejorar los desenlaces clínicos, la seguridad del paciente y disminuir el costo de atención en las instituciones de salud; aunque la diminución de estos grupos es la tendencia. El objetivo de este artículo es presentar la información acerca del estado y progreso de los grupos de soporte nutricional en Colombia y en el mundo obtenida en el nutritionDay en el periodo comprendido entre 2011 y 2016.

Metodología: el nutritionDay es una auditoría internacional de corte transversal, aplicada en un día y a todo tipo de servicios hospitalarios. Los datos se recopilan con la ayuda de cinco cuestionarios y sus resultados se presentan en porcentaje.

Resultados: el porcentaje de instituciones que cuenta con grupo de soporte nutricional multidisciplinario en Colombia varía entre $47 \%$ y $65 \%$, mientras que entidades internacionales reportan entre $72 \%$ y $82 \%$. En 2016, 82,5\% de las instituciones colombianas tenían una estrategia de cuidado nutricional comparado con $72 \%$ en el ámbito internacional.

Conclusión: la encuesta del nutritionDay (2011 - 2016) permite conocer la evolución de los grupos de soporte nutricional y la estrategia de cuidado nutricional que realizan las instituciones colombianas participantes. Se recomienda realizar un estudio nacional que permita caracterizar el funcionamiento de los grupos en Colombia.
\end{abstract}

Palabras clave: apoyo nutricional, terapia nutricional, desnutrición, grupos de soporte nutricional.

1 Instituto de Investigación en Nutrición, Genética y Metabolismo, Facultad de Medicina, Universidad El Bosque, Bogotá, D.C., Colombia.

2 Servicio de Cirugía, Hospital de Meissen, Bogotá, D.C., Colombia.

\section{Summary}

Background: Nutritional care through interdisciplinary nutritional support groups and under standards and clinical guidelines has been shown to improve patient outcomes and safety with a positive financial impact on health institutions. However, the world tendency is to the diminution of these groups. The objective of this article is to present the information about the status and evolution of the nutritional support groups in Colombia obtained in the nutritionDay in the period 2011 to 2016.

Methodology: nutritionDay is an international cross-sectional audit, in one day and in all types of hospital services. The data is collected with five questionnaires. The results are presented as percentage.

Results: the percentage of institutions with a multidisciplinary nutritional support group in Colombia varies between 47 $\%$ and $65 \%$, while at an international level it varies between $72-$ $82 \%$. In 2016, $82.5 \%$ of Colombian institutions had a nutritional care strategy compared to $72 \%$ internationally.

Conclusion: the nutritionDay survey between 2011 and 2016 allows us to know the evolution of the nutritional support groups and the nutritional care strategy carried out by the participating Colombian institutions. It is recommended to carry out a national study to know thoroughly the functioning of the groups in Colombia.

Keywords: Nutritional support teams; Malnutrition; Nutritional care; Nutrition Support Practice.

3 Grupo de Soporte Nutricional, Clínica del Country, Clínica VIP; Grupo de Soporte Nutricional, Clínica La Colina, Bogotá, D.C., Colombia. 


\section{INTRODUCCIÓN}

La desnutrición asociada a la enfermedad es un problema prioritario de salud pública en el mundo. En Latinoamérica, alrededor de $50 \%$ de los pacientes adultos en los hospitales de Argentina, Brasil, Chile, Costa Rica, Cuba, República Dominicana, Ecuador, México, Panamá, Paraguay, Perú, Puerto Rico, Venezuela y Uruguay tienen algún grado de desnutrición ${ }^{(1-7)}$. La desnutrición tiene un impacto importante sobre la morbimortalidad, la calidad de vida, la estancia hospitalaria y los costos en salud ${ }^{(8,9)}$.

Con el desarrollo de la terapia nutricional especializada (nutrición enteral, parenteral y suplementos orales), ha sido esencial un enfoque interdisciplinario para traducir este avance científico del "laboratorio a la cabecera del paciente". La historia muestra que a medida que se adoptó esta innovación, se crearon equipos interdisciplinarios de terapia o soporte nutricional para optimizar la efectividad y la seguridad del cuidado nutricional $^{(10)}$. El impacto de la estandarización y el uso de un equipo interdisciplinario para proporcionar esta terapia han demostrado mejorar los resultados y la seguridad del paciente con un impacto financiero positivo en las instituciones de salud ${ }^{(11-13)}$.

La estructura y la función de los equipos interdisciplinarios de soporte nutricional varían de una institución a otra según las necesidades locales, la cultura organizacional y el personal disponible. La composición ideal del equipo incluye médicos, enfermeras, nutricionistas y farmacéuticos con formación especializada en nutrición. Los terapeutas y rehabilitadores físicos también cumplen un papel importante y varios autores reconocen la necesidad de incluirlos ${ }^{(14)}$. Si no existe un equipo interdisciplinario, los procesos del cuidado nutricional (tamizaje, diagnóstico, plan de cuidado nutricional, terapia nutricional, etc.) deben ser realizadas de manera individual por profesionales de distintas disciplinas, quienes pueden o no ser competentes para realizar las funciones adecuadamente ${ }^{(8)}$.

El equipo interdisciplinario es el encargado de que se cumpla a cabalidad el proceso de cuidado nutricional $y$ entre sus funciones se incluyen: realizar el tamizaje y la evaluación nutricional, la determinación del diagnóstico y la severidad de la desnutrición, la determinación de las necesidades de energía, proteína y micronutrientes, establecer la indicación de nutrición enteral/parenteral, la evaluación del acceso a la terapia nutricional, monitoreo, evaluación y documentación de la terapia nutricional $^{(8)}$
Sin embargo, muchos hospitales en Latinoamérica $y$ en el mundo no cuentan con equipos de soporte nutricional, y, es más, la cantidad parece estar disminuyendo ${ }^{(15,16)}$. Para ser eficaces los equipos de soporte nutricional deben realizar una práctica basada en la evidencia y medir su desempeño.

Colombia ha participado en el nutritionDay, diseñada y promovida por la Sociedad Europea de Nutrición Clínica y Metabolismo (ESPEN) desde el año 2009, que permite conocer la organización del cuidado nutricional hospitalario, además de promover la importancia de un adecuado cuidado nutricional para luchar contra la desnutrición hospitalaria ${ }^{(17)}$. El objetivo de este artículo es presentar la información acerca del estado y progreso de los grupos de soporte nutricional a través del nutritionDay 2011-2016 en Colombia y en el ámbito internacional.

\section{METODOLOGÍA}

El nutritionDay es una auditoría internacional de corte transversal, aplicada en un día y a todo tipo de servicios hospitalarios (medicina interna, cirugía, unidad de cuidado intensivo, etc); también incluye todos los pacientes presentes en la unidad durante el día del estudio. Se excluyen los pacientes menores de 18 años, ambulatorios, hospitalización programada $<24$ horas y quienes se nieguen a participar.

Los datos del nutritionDay se recopilan mediante cinco cuestionarios y para esta investigación se tomó la información de número de pacientes, número de unidades, edad y sexo; también se tuvieron en cuenta las siguientes preguntas:

1. ¿La institución cuenta con un grupo de soporte nutricional? y las preguntas introducidas en 2016:

2. ¿Tiene la unidad una estrategia de cuidado nutricional?

3. ¿Utiliza rutinariamente pautas o estándares para cuidado nutricional?

La fuente de información para la presente investigación proviene de los reportes nacionales para Colombia 2011-2016. (nutritionDay National Reports: Disponible en https://goo.gl/LGCsak. Consultado el 13 junio, 2018). El análisis estadístico del nutritionDay fue realizado por Department for Medical Statistics, Medical University of Vienna. Los resultados en el presente artículo se reportan en porcentajes.

El estudio nutritionDay cuenta con la aprobación del Comité de Ética de la Universidad de Viena. En Colombia, tuvo la aprobación del Comité Institucional 
de Ética de la Universidad El Bosque, y el respaldo de la Asociación Colombiana de Nutrición Clínica. Cada institución participante recibió la aprobación del comité correspondiente.

\section{RESULTADOS}

Colombia aportó al nutritionDay el 12,7 \% (10.396) de los pacientes y el 8,6 \% (342) de las unidades (servicios hospitalarios) en el periodo de estudio. La edad promedio (rango) de los pacientes fue 62 años (12103), $48 \%$ de los encuestados eran del sexo femenino. En Colombia, y en el mundo, las principales unidades participantes por especialidad, a lo largo de los años, fueron medicina interna y cirugía.

El porcentaje de instituciones que cuenta con grupo de soporte nutricional multidisciplinario constituido en Colombia varía entre $47 \%$ y $65 \%$, mientras que el referente internacional fluctúa entre $72 \%$ y $82 \%$. En la Figura 1 se muestra el porcentaje de instituciones con grupo de soporte nutricional en Colombia en comparación con el resto del mundo.

En 2016, 82,5\% de las instituciones de Colombia tenían una estrategia de cuidado nutricional comparado con $72 \%$ de entidades internacionales. La utilización rutinaria de pautas o estándares para cuidado nutricional en Colombia se muestra en la Figura 2.

\section{DISCUSIÓN}

Los resultados obtenidos de la participación de Colombia en el nutritionDay durante el periodo 20112016 demuestran que Colombia tiene un menor porcentaje de equipos de soporte nutricional en comparación con el resto del mundo; a pesar de que en Colombia, diferentes instituciones entre ellas la Fundación Santa Fe de Bogotá, el Hospital Militar Central, el Hospital San Ignacio, y la ACNC, promueven la calidad del cuidado nutricional, y se ha logrado incrementar el número de hospitales participantes en el nutritionDay 2016, la cantidad de grupos de soporte nutricional no aumenta. Es de resaltar que los hallazgos pueden subestimar la realidad del país, debido a que la participación en el nutritionDay es voluntaria, lo que genera la posibilidad de que las instituciones más sensibilizadas con el tema, y por lo tanto con un grupo ya constituido, son las que participan mayoritariamente en la encuesta.

Sin embargo, la tendencia a la disminución en la cantidad de grupos de soporte nutricional es universal. En una encuesta de la Sociedad Americana de Nutrición Enteral y Parenteral (ASPEN por sus siglas en inglés), con 698 respuestas parciales y 200 completas, $42 \%$ de las instituciones realizan soporte nutricional en equipo de soporte nutricional ${ }^{(15,16)}$ y se ha mantenido estable en las instituciones norteamericanas en las últimas tres décadas.

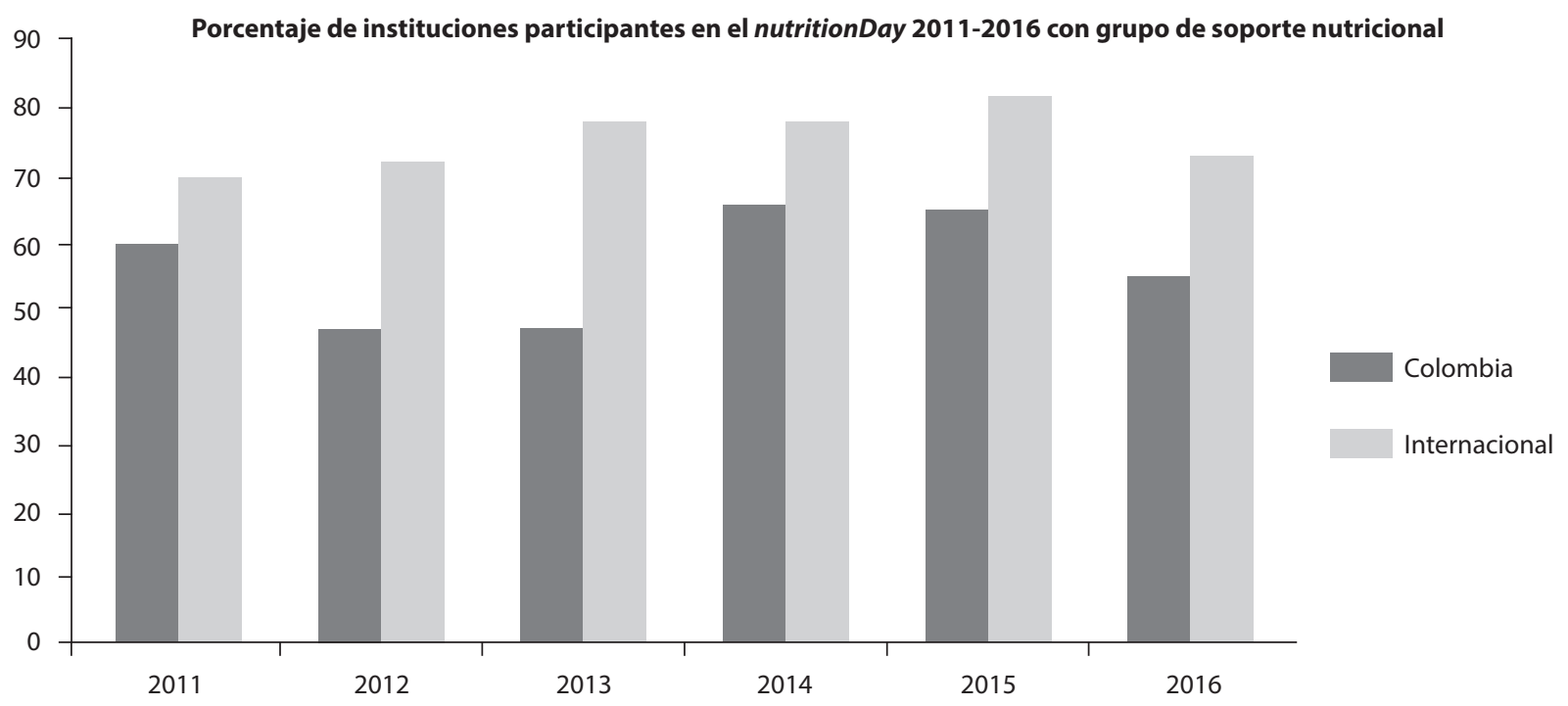

Figura 1. Instituciones participantes en el nutritionDay 2011-2016 con grupo de soporte nutricional. 


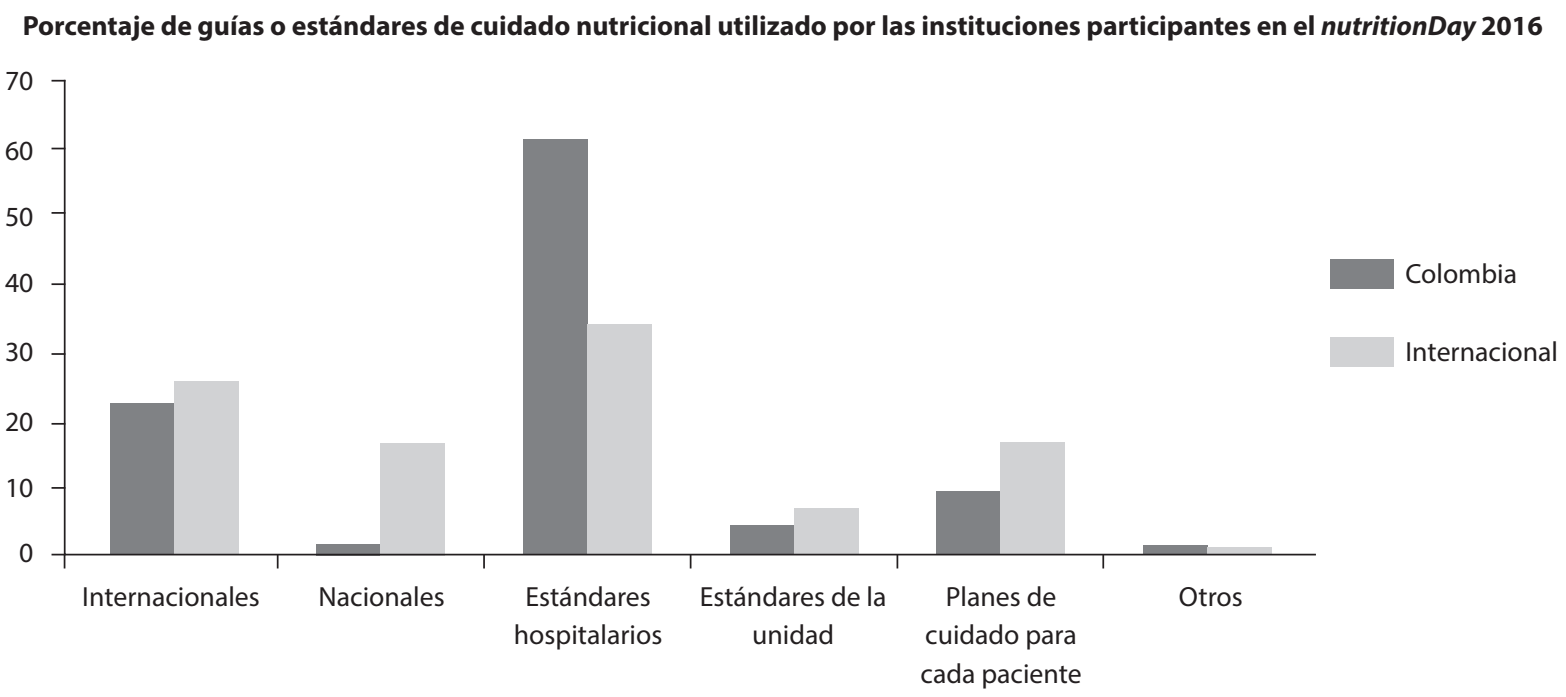

Figura 2. Utilización rutinaria de guías o estándares de cuidado nutricional en Colombia, según datos obtenidos en el nutritionDay 2016.

La encuesta de ASPEN mostró que $23 \%$ de las instituciones sin grupo, antes tenían uno, y las razones más comunes para no tener un grupo de soporte incluyen servicios de apoyo nutricional descentralizados (24\%), falta de interés de liderazgo médico (23\%), problemas económicos (17\%), falta de apoyo administrativo (12 $\%)$ y falta de tiempo $(10 \%)^{(15)}$. Además, $22 \%$ de las instituciones sin grupo de soporte, que anteriormente tenían, afirmaron que los resultados de los pacientes se han visto afectados en forma negativa por este cambio.

En Europa, varios estudios han demostrado que la presencia de grupos de soporte nutricional varía ampliamente de un país a otro, pero de manera general son esca$\operatorname{sos}^{(18,19)}$. Por ejemplo, S Klerk, et al. en una muestra de 160 hospitales de Grecia y Estonia encontraron que no hay grupos de soporte, mientras que en Polonia, $72 \%$ de las instituciones contaban con un grupo ${ }^{(17)}$.

La actividad clínica asistencial se rige por guías y protocolos nacionales e internacionales. La implementación de estas guías basadas en evidencia es fundamental para el éxito de los equipos interdisciplinarios y la realización del soporte nutricional apropiada ${ }^{(15)}$. Como lo describen Delegge, et al ${ }^{(15)}$, se deben tener en cuenta muchos aspectos para implementar con éxito estas guías en el entorno clínico, entre ellos: la conciencia de la existencia de las guías, consenso entre los profesionales sobre las pautas que se deciden adoptar $y$ una adherencia de los profesionales a dichas guías. En Colombia, $82,5 \%$ de las unidades tienen una estrategia de cuidado nutricional, $60,9 \%$ utiliza estándares hospitalarios y $21,9 \%$ guías internacionales.

El cuidado nutricional utilizando estándares de práctica y guías de manejo a través de un equipo interdisciplinario de soporte nutricional ha demostrado mejorar los resultados clínicos, la seguridad del paciente y un impacto financiero positivo en las instituciones de salud. Con los grupos de soporte nutricional, es más probable que se cumplan los requerimientos de energía de los pacientes y se reduzcan las complicaciones mecánicas y metabólicas de la terapia nutricional ${ }^{(20-25)}$. Los autores de un metanálisis de estudios publicados entre 1970 y $1993^{(26)}$ concluyeron que los equipos de soporte nutricional reducen la tasa de infección relacionada con catéter y complicaciones metabólicas, mejoran la documentación y, probablemente, también reducen los costos, aunque los costos de personal del equipo no fueron tomados en consideración en todos los estudios.

Los beneficios económicos de establecer un equipo de apoyo nutricional, e incluso de una enfermera especializada en nutrición, han sido ampliamente documentados desde la década de los $90^{(27-30)}$. La estandarización de la selección de los productos nutricionales y de la prescripción puede generar ahorros significativos ${ }^{(31)}$.

Las actividades de los equipos de soporte nutricional en Colombia se realizan tradicionalmente en tres grandes campos: educación e investigación, y asistencia. Otros autores han descrito las funciones estándar de los equipos de soporte nutricional. Sabino define 
los objetivos de los grupos en Colombia, los cuales se han mantenido desde los años 80. Estos objetivos se traducen en funciones clínicas, didácticas y de investigación ${ }^{(32,33)}$. Lenzi et al. mencionan desde los años 90 , que tradicionalmente los grupos de soporte metabólico y nutricional cubren las funciones de interconsulta, programas de educación, investigación, programas de calidad y nutrición ambulatoria ${ }^{(34)}$. A pesar de lo anterior, el nutritionDay no aborda este tema y podría ser un tema de investigación en el futuro.

\section{CONCLUSIÓN}

La encuesta del nutritionDay entre 2011 y 2016 permite conocer la evolución de los grupos de soporte nutricional y la estrategia de cuidado nutricional que realizan las instituciones colombianas participantes. La mayoría de las instituciones tiene una estrategia de cuidado nutricional con guías y estándares institucionales. Se recomienda realizar un estudio nacional para conocer a fondo el funcionamiento de los grupos en Colombia.

\section{Agradecimientos}

A la Asociación Colombiana de Nutrición Clínica y Junta Directivas y a los coordinadores nacionales por el apoyo y compromiso en la realización del NutritionDay en Colombia.

\section{Referencias bibliográficas}

1. Correia MI, Hegazi RA, Diaz-Pizarro Graf José Ignacio, Gomez-Morales G, Fuentes Gutiérrez C, Goldin MF, Navas A, Pinzón Espitia OL, a Millere Tavares G. Addressing DiseaseRelated Malnutrition in Healthcare: A Latin American Perspective. JPEN J Parenter Enteral Nutr. 2016;40:319-25.

2. Correia MI, Campos AC. Prevalence of hospital malnutrition in Latin America: the multicenter ELAN study. Nutrition. 2003;19(10):823-5.

3. Wyszynski DF, Perman M, Crivelli A. Prevalence of hospital malnutrition in Argentina: preliminary results of a populationbased study. Nutrition. 2003;19(2):115-9.

4. Veramendi-Espinoza LE, Zafra-Tanaka JH, Salazar-Saavedra $\mathrm{O}$, et al. Prevalence and associated factors of hospital malnutrition in a general hospital; Peru, 2012 [in Spanish]. Nutr Hosp. 2013;28(4):1236-1243. 15.

5. Waitzberg DL, Caiaffa WT, Correia MI. Hospital malnutrition: the Brazilian national survey (IBRANUTRI): a study of 4000 patients. Nutrition. 2001;17(7-8):573-80.

6. Gallegos Espinosa S, Nicolalde Cifuentes M, Santana Porben S. State of malnutrition in hospitals of Ecuador. Nutr Hosp. 2014;30(2):425-35.
7. Giraldo Giraldo NA, Múnera García NE, Espitaleta Marrugo V, Pinerez LM. Prevalence of malnutrition and evaluation of dietary treatment for adult hospitalized patients in a public institution of high complexity. Perspect Nut Hum. 2007;9:37-47.

8. Curtis LJ, Bernier P, Jeejeebhoy K, Allard J, Durksen D, Gramlich L, Laporte M, Keller HH. Cost of hospital malnutrition. Clin Nutr. 2017;36:1391-6.

9. Lim SL, Ong KC, Chan YH, Loke WC, Ferguson M, Daniels L. Malnutrition and its impact on cost of hospitalization, length of stay, readmission and 3-year mortality. Clin Nutr. 2012;3:345-50.

10. Schneider PJ. Nutritition support teams: an evidence-based practice. Nutr Clin Pract. 2006; 21(1): 62-7.

11. Boitano M, Bojak S, McCloskey S, McCaul DS, McDonough M. Improving the safety and effectiveness of Parenteral Nutrition: results of a quality improvement collaboration. Nutr Clin Pract. 2010;25:663-71.

12. Schneider PJ. Nutritition support teams: an evidence-based practice. Nutr Clin Pract. 2006;21:62-7.

13. Bischoff SC, Kester L, Meier R, Radziwill R, Schwab D, Thul P. Organization, regulations, preparation and logistics of parenteral nutrition in hospital and homes; the role of the nutrition support team-Guidelines on Parenteral Nutrition, Chapter 8. GMS Ger Med Sci. 2009;7:1-8.

14. Martin A. La formation à la nutrition des professionnels, Rapport au Ministre de la santé, Avril 2009. Disponible en: http://socialsante.gouv.fr/IMG/pdf/Rapport_A_Martin_ formation_nutrition.pdf. Consultado el 20 de mayo 2018.

15. DeLegge $\mathrm{MH}$, Kelly AT. State of nutrition support teams. Nutr CLin Pract. 2013;28(6):691-7.

16. DeLeggeM, Wooley JA, Guenter P, Wright Sh, Brill J, Andris D, Wagner P, Filibeck D, and A.S.P.E.N. Board of Directors. The State of Nutrition Support Teams and Update on Current Models for Providing Nutrition Support Therapy to Patients. Nutr Clin Pract. 2010;25:76-84.

17. Schindler K, Pichard C, Sulz I, Volkert D, Streicher M, Singer P, et al. nutritionDay: 10 years of growth. Clin Nutr. 2017;36:1207-14.

18. Klek S, Krznaric Z, Gundogdu RH, Chourdakis M, Kekstas G, Jakobson T, et al. Prevalence of malnutrition in various political, economic, and geographic settings. JPEN J Parenter Enteral Nutr. 2015;39:200-10.

19. Shang E, Hasenberg T, Schlegel B, Sterchi AB, Schindler K, Druml W, Koletzko B, Meier R. An European survey of structure and organization of nutrition support teams in Germany, Austria and Switzerland Clin Nutr. 2005;24(6):1005-13.

20. Brown RO, Carlson SD, Cowan GS Jr, Powers DA, Luther RW. Enteral nutritional support management in a university teaching hospital: team vs nonteam. JPEN J Parenter Enteral Nutr. 1987;11:52-6.

21. Chrisanderson D, Heimburger DC, Morgan SL, Geels WJ, Henry KL, Conner W, et al. Metabolic complications of total 
parenteral nutrition: effects of a nutrition support service. JPEN J Parenter Enteral Nutr. 1996;20:206-10.

22. Dalton MJ, Schepers G, Gee JP, Alberts CC, Eckhauser FE, Kirking DM. Consultative total parenteral nutrition support teams: the effect on the incidence of total parenteral nutrition-related complications. JPEN J Parenter Enteral Nutr. 1984;8:146-52.

23. Fettes SB, Lough M. An audit of the provision of parenteral nutrition in two acute hospitals: team versus non-team. Scott Med J. 2000;45(4):121-5.

24. Gales BJ, Riley DG. Improved total parenteral nutrition therapy management by a nutritional support team. Hosp Pharm.1994;29:469-70.

25. Traeger SM, Williams GB, Milliren G, Young DS, Fisher M, Haug MT 3rd. Total parenteral nutrition by a nutrition support team: improved quality of care. JPEN J Parenter Enteral Nutr. 1986;10:408-12.

26. Gales BJ, Gales MJ. Nutritional support teams: a review of comparative trials. Ann Pharmacother. 1994;28(2):227-35.

27. Gianino MS, Brunt LM, Eisenberg PG. The impact of a nutritional support team on the cost and management of multilumen central venous catheters. J Intraven Nurs. 1992;15(6):327-32.
28. Trujillo EB, Young LS, Chertow GM, et al. Metabolic and monetary costs of avoidable parenteral nutrition use. JPEN J Parenter Enteral Nutr. 1999;23(2):109-13.

29. Goldstein M, Braitman LE, Levine GM. The medical and financial costs associated with termination of a nutrition support nurse. JPEN J Parenter Enteral Nutr. 2000;24:323-7.

30. Trujillo EB, Young LS, Chertow GM, et al. Metabolic and monetary costs of avoidable parenteral nutrition use. JPEN J Parenter Enteral Nutr. 1999;23:109-113.

31. Maswoswe JJ, Newcomer DR, Quandt CM. Achieving parenteral nutrition cost savings through prescribing guidelines and formulary restrictions. Am J Hosp Pharm. 1987;44(6):1376-81.

32. Sabino P. Desnutrición hospitalaria: grupos de soporte metabólico y nutricional. Rev. Colomb. Cir. 2012;27:46-54.

33. Hamaqui E, Rombeau J. The nutrition support team. En: Rombeau J, Caldwell M, Editors. Parenteral Nutrition. Philadelphia: W. B. Saunders Company; 1986.

34. Lenzi M. Surviving and thriving in an era of freedom. Surviving and thriving in an era of reform. Nutr Clin Pract. 1995; 10:17s-23s. 